\title{
Using ACL2 in the Design of Efficient, Verifiable Data Structures for High-Assurance Systems*
}

\author{
David Hardin ${ }^{\dagger}$ \\ Advanced Technology Center \\ Rockwell Collins \\ Cedar Rapids, IA USA \\ david.hardin@rockwellcollins.com
}

\author{
Konrad Slind \\ Advanced Technology Center \\ Rockwell Collins \\ Bloomington, MN USA \\ konrad.slind@rockwellcollins.com
}

\begin{abstract}
Verification of algorithms and data structures utilized in modern autonomous and semi-autonomous vehicles for land, sea, air, and space presents a significant challenge. Autonomy algorithms, e.g., route planning, pattern matching, and inference, are based on complex data structures such as directed graphs and algebraic data types. Proof techniques for these data structures exist, but are oriented to unbounded, functional realizations, which are not typically efficient in either space or time. Autonomous systems designers, on the other hand, generally limit the space and time allocations for any given function, and require that algorithms deliver results within a finite time, or suffer a watchdog timeout. Furthermore, high-assurance design rules frown on dynamic memory allocation, preferring simple array-based data structure implementations.

In order to provide efficient implementations of high-level data structures used in autonomous systems with the high assurance needed for accreditation, we have developed a verifying compilation technique that supports the "natural" functional proof style, but yet applies to more efficient data structure implementations. Our toolchain features code generation to mainstream programming languages, as well as GPU-based and hardware-based realizations. We base the Intermediate Verification Language for our toolchain upon higher-order logic; however, we have used ACL2 to develop our efficient yet verifiable data structure design. ACL2 is particularly well-suited for this work, with its sophisticated libraries for reasoning about aggregate data structures of arbitrary size, efficient execution of formal specifications, as well as its support for "single-threaded objects" - functional datatypes with imperative "under the hood" implementations.

In this paper, we detail our high-assurance data structure design approach, including examples in ACL2 of common algebraic data types implemented using this design approach, proofs of correctness for those data types carried out in ACL2, as well as sample ACL2 implementations of relevant algorithms utilizing these efficient, high-assurance data structures.
\end{abstract}

\section{Introduction}

As autonomous systems have matured from laboratory curiosities to sophisticated platforms poised to share our roadways, sea lanes, and airspace, accrediting agencies are faced with the significant challenge of verifying and validating these systems to ensure that they do not constitute a significant societal risk. Leaving aside the issues with the verification and validation of deep learning, even basic autonomy algorithms, e.g., route planning, pattern matching, and inference, are based on complex data structures, such as directed graphs and algebraic data types. Proof techniques for these data structures exist, but are oriented to unbounded, functional realizations, which are not typically efficient in either space or time.

\footnotetext{
*Approved for Public Release, Distribution Unlimited

$\dagger$ The views expressed are those of the authors and do not reflect the official policy or position of the Defense Advanced Research Projects Agency (DARPA) or the U.S. Government.
}

Shilpi Goel and Matt Kaufmann (Eds.):

ACL2 Theorem Prover and its Applications (ACL2-2018)

EPTCS 280, 2018, pp. 61-76, doi:10.4204/EPTCS.280.5 
Autonomous systems designers, on the other hand, generally limit the space and time allocations for any given function, and require that algorithms deliver results within a finite time, or suffer a watchdog timeout. Furthermore, high-assurance design rules, such as mandated by RTCA DO-178C Level A [24] for flight-critical systems, frown on dynamic memory allocation, preferring simple array-based data structure implementations.

In order to provide efficient implementations of high-level data structures used in autonomous and other critical systems with the high assurance needed for accreditation, we have been developing a verification technique that supports the "natural" functional proof style, but yet applies to more efficient data structure implementations. We have used ACL2 to prototype and refine an in-place data structure representation amenable for formal proof, as detailed in Section 6 . Applications include path planning on high-level graphs, inference engines for autonomous mission executives, etc.

We have significant experience in the formal verification of practical engineering artifacts [7]. Our particular experience with the formal verification of array-based implementation of algebraic data types indicates that these proofs are extremely difficult, whether one takes a theorem proving [9], model checking [10], or symbolic execution [2] approach. The former approach, while yielding a proof for arbitrary array size, easily gets bogged down in supplementary details (even when using list/array "bridging" constructs such as ACL2 single-threaded objects [3]), whereas the latter two approaches suffer from lack of scalability, leading to timeouts except for very small arrays — but unsurprisingly, most practical algorithms operate routinely on thousands of data elements. Therefore, we need a different approach.

We have applied verifying compiler technology to this problem. Algorithms are expressed in a Domain-Aware System Language. A Domain-Aware language is one whose features are informed by the requirements of the domain, such as primitive operations, environmental constraints, assurance and accreditation requirements, and so on, but which is not overtly tied to a particular domain. Our primary goal is to craft a verification-enhanced programming language to support the high assurance requirements of the domains in which it will be used.

\section{Verification-Enhanced Programming Languages}

A verification-enhanced programming language is one in which properties of programs in the language can be formally stated and reasoned about in an integrated environment. Examples of such languages are SPARK/Ada [19], Dafny [16], Guardol [12], and certain C dialects ${ }^{1}$ [18 27]. Reasoning environments for $\mathrm{C}$ have become highly developed because of the pervasive usage of $\mathrm{C}$ in important system infrastructure such as operating systems, cryptography libraries, etc.; but $\mathrm{C}$ does not support our goal of producing high-level functional correctness proofs.

Once one has constructed a verification-enhanced language, it is relatively simple to create another: after a point, much of the middle and backend processing changes little, leaving only the frontend to be adjusted to the new concrete syntax and type system. This explains the rise of the intermediate verification language. For example, Boogie [17] underlies a number of languages, such as VCC and Dafny, and WhyML [5] underlies verification-enhanced versions of C and Ada. Figure 1 illustrates the basic toolchain pattern: an IDE is used to create and edit programs and their specifications; typechecking and semantic checking of programs usually takes place here as well, in order to give good feedback on program construction. The program is then mapped to an abstract syntax tree (AST) representation suitable for code generation/compilation or for mapping into a verification-friendly format. Source-to-

\footnotetext{
${ }^{1}$ Note that any logic capable of expressing computable functions, e.g., ACL2 [14 $\mid$ or higher order logic, can be regarded as a verification-enhanced programming language; our emphasis here is on more conventional programming languages.
} 


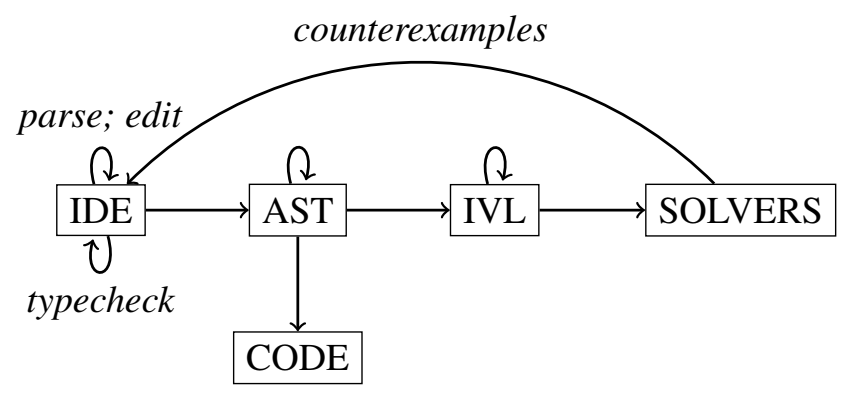

Figure 1: Verification tool design pattern.

source transformations are possible in the AST representation and also in the IVL representation $\sqrt{2}$ From the IVL representation, SMT solvers, or other automatic methods can be invoked. Interactive proofs can also be initiated for properties lying outside the domain of automation. In case the backend solvers provide counterexamples, they may be translated into user-friendly format and passed back to the IDE.

We note the following benefits of providing the IVL with a formal operational semantics:

1. The semantics provide a formal basis for individual programs to be proved correct. By use of decompilation into logic [20], imperative programs in the operational semantics can be converted to equivalent logic functions by deduction.

2. The formal semantics are the basis for proving the correctness of AST-to-AST transformations.

3. An exciting aspect of the CakeML verified compiler [15] is the existence of a verifying translation from entities in the HOL logic to CakeML programs [21]. As a consequence, HOL functions can be defined and have properties proved about them, then can be automatically translated to CakeML. The correctness of the CakeML compiler ensures that the behavior of the compiled binary version of the function is that of the original logic function. We can further leverage this capability to establish a formal connection between the AST for the original program and the final executable.

\section{Modelling Imperative Languages}

We will use DASL as an example of a verification-enhanced language. DASL (Domain-Aware System Language) is a first order hybrid functional/imperative language with constructs familiar from Ada, ML, Swift and other such languages. The constructible types build on a standard collection of base types: booleans, signed and unsigned integers (both bounded and unbounded), characters, and strings. Arrays and records support aggregation and ML-style recursive datatypes provide tree-shaped data. Programs are built from assignment, sequencing, conditional statements, and (possibly recursive) procedures. The main novelty in the statement language is support for ML-style match statements over the construction of datatypes. The concrete syntax is conventional and should not cause any surprises. The instantiation of the verification tool architecture of Figure 1 to DASL is shown in Figure 2.

We chose HOL4 as the environment for the "middle-end" of the DASL compilation toolchain due to its support for higher-order logic, the primary DASL developer's HOL4 expertise, as well as the verified path from executable logic function to binary code provided by the HOL4-based CakeML toolchain.

\footnotetext{
${ }^{2}$ In some systems, the AST representation and the IVL representation coincide.
} 


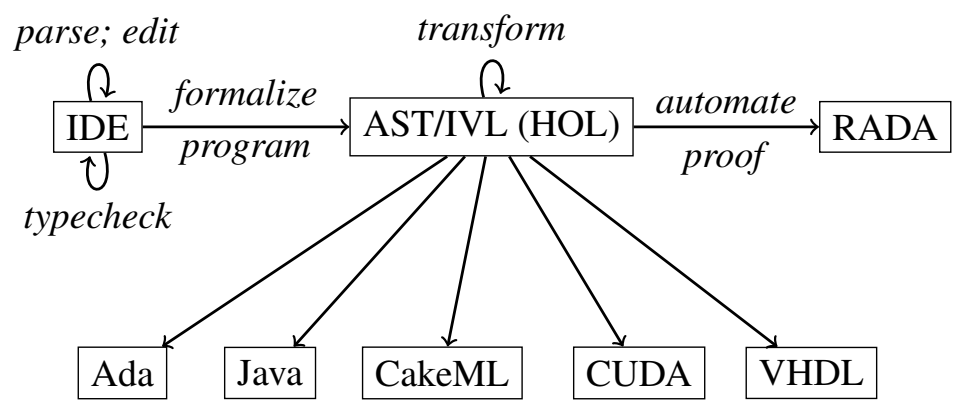

Figure 2: DASL toolchain.

Once in the AST format, source code can be generated for a variety of programming languages. The backend solver is RADA, a SMT-based system for reasoning about recursive programs over algebraic datatypes [23].

\subsection{Formal Operational Semantics}

The operational semantics of DASL describes program evaluation by a 'big-step' inductively defined judgement saying how statements alter the program state. The formula STEPS $\Gamma$ code $s_{1} s_{2}$ says "evaluation of statement code in environment $\Gamma$ beginning in state $s_{1}$ terminates and results in state $s_{2}$ ". We have also formalized a small-step semantics and proved equivalence of the two semantics. Note that $\Gamma$ is an environment binding procedure names to procedure bodies. We follow an approach taken by Norbert Schirmer [25], wherein he constructed a generic semantics capturing a large class of sequential imperative programs, and then instantiated the generic semantics to a given programming language.

\subsection{Decompilation into Logic}

The pioneering work of Myreen [20] introduced the idea of decompiling assembly programs to higher order logic functions; we have adapted his approach to our high-level imperative language. For us, a decompilation theorem has the stylized form

$$
\begin{aligned}
& \vdash \forall s_{1} s_{2} . \forall x_{1} \ldots x_{k} . \\
& \quad s_{1} . \text { proc. } v_{1}=x_{1} \wedge \cdots \wedge s_{1} . \text { proc. } v_{k}=x_{k} \wedge \\
& \quad \text { STEPS } \Gamma \text { code }\left(\text { Normal } s_{1}\right)\left(\text { Normal } s_{2}\right) \\
& \quad \Rightarrow \\
& \quad \text { let }\left(o_{1}, \ldots, o_{n}\right)=f\left(x_{1}, \ldots, x_{k}\right) \\
& \quad \text { in } s_{2}=s_{1} \operatorname{with}\left\{\text { proc. } w_{1}:=o_{1}, \ldots, \text { proc. } w_{n}:=o_{n}\right\}
\end{aligned}
$$

which essentially states that evaluation of code implements HOL function $f$. The antecedent $s_{1}$.proc. $v_{1}=$ $x_{1} \wedge \cdots \wedge s_{1}$. proc. $v_{k}=x_{k}$ binds logic variables $x_{1} \ldots x_{k}$ to the values of program variables $v_{1} \ldots v_{k}$ in state $s_{1}$. These values form the input for the so-called footprint function $f$, which delivers the output values $o_{1}, \ldots, o_{n}$ that are used to update $s_{1}$ to $\left.s_{2}\right]^{3}$ One can see that a decompilation theorem is a particular kind of Hoare triple. (An explicit Hoare triple approach is used by Myreen in his work.)

\footnotetext{
${ }^{3}$ In our modelling, a program state is represented by a record containing all variables in the program. The notation s.proc.v denotes the value of program variable $v$ in procedure proc in state $s$. The with-notation represents record update.
} 
NB. The footprint function $f$ is automatically synthesized from code and the decompilation theorem is proved automatically. In other words, decompilation is an algorithm: it always succeeds, provided that all footprint functions arising from the source program terminate.

Decompilation can also be applied to the problem of creating goals from program specifications. A DASL specification sets up a computational context—a state—and asserts that a property holds in that state. In its simplest form, a specification looks like

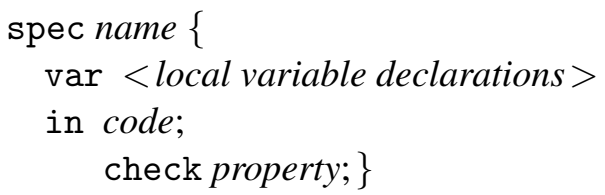

where property is a boolean expression. A specification declaration is processed as follows. First, suppose that execution of code starts normally in $s_{1}$ and ends normally in $s_{2}$, i.e., assume

STEPS $\Gamma$ code $\left(\right.$ Normal $\left.s_{1}\right)\left(\right.$ Normal $\left.s_{2}\right)$.

We want to show that property holds in state $s_{2}$. We decompile code to footprint function $f$ and decompile property to footprint function $g$; then, formally, we need to show

$$
\begin{aligned}
& \left(\text { let }\left(o_{1}, \ldots, o_{n}\right)=f\left(x_{1}, \ldots, x_{k}\right)\right. \\
& \text { in } \left.s_{2}=s_{1} \text { with }\left\{\text { name. } w_{1}:=o_{1}, \ldots, \text { name. } w_{n}:=o_{n}\right\}\right) \\
& \Rightarrow g s_{2}
\end{aligned}
$$

The proof proceeds using facts about $f$, principally its recursion equations and induction theorem, to show the translated property $g$ holds on values projected from the final state $s_{2}$. The original code and property have been freed—by sound deductive steps—-from the program state and operational semantics.

\section{Data Structure Compilation}

Compilation takes a DASL package expressed as a collection of type and procedure declarations, and maps it, when a fixed size declaration is present, to another package where algebraic datatypes have been replaced by array-based representations, and functions over the datatypes have been similarly lowered.

The compilation of data structures is phrased as a source-to-source translation on DASL ASTs. It bears some resemblance to the compiler described in [26]. The target representation was originally used in graph algorithms on GPUs, but also serves quite well for imperative algebraic datatypes.

\subsection{Target Representation}

Our array-based data structure representation is adapted from work by Harish and Narayanan [13] on efficient graph algorithms for GPUs using the CUDA language; this layout was previously ported to ACL2 single-threaded objects [9]. This design supports graphs with data at vertices and also on edges. Graphs are bounded: both the number of vertices and the number of outgoing edges per vertex are bounded.

One modification we have made to the layout described in the Harish and Narayanan paper [13] is that zero designates a null vertex index or null edge index. We explicitly allocate a zeroth element for each array, and prove that the zeroth elements are unchanged by any array mutator. 


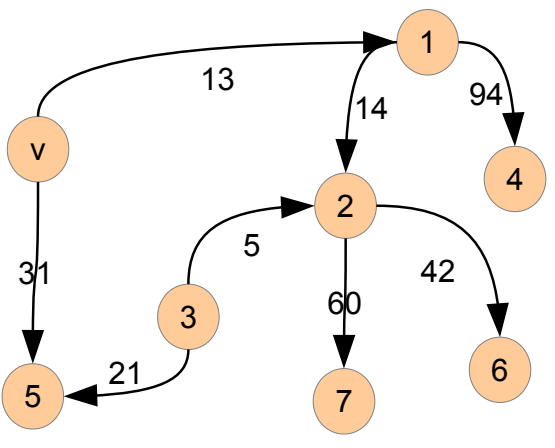

(a)

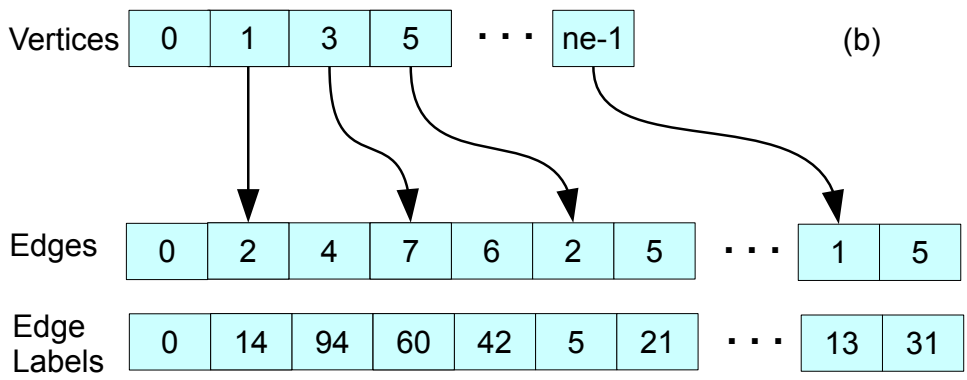

Figure 3: (a) Graph fragment with two edges per vertex; (b) Array-based layout for the graph fragment.

A graph with maximum number of vertices $N$ and maximum number of outgoing edges per vertex $M$ is represented by a seven-tuple store $=(V, D, E, W, V h d, V t l, V$ count $)$ where

- $V$ is the vertex array of length $N+1$;

- $D$ is the data associated with each vertex, requiring an array of length $N+1$;

- $E$ is the edge array of length $M N+1$;

- $W$ holds the "weight" or "label" data associated with each edge, also an array of length $M N+1$;

- Vhd is the index of the "head" vertex in $V$;

- Vtl is the index of the "tail" vertex in $V$; and

- Vcount is the number of non-zero elements in $V$.

The vertex array contains indices into the edge array, whereas the edge array contains vertex indices, as shown in Fig. 3 (the data associated with each vertex are not shown, in the interest of clarity). The weight array contains the weight of each edge, and thus is the same size as the edge array. Note that this basic graph tuple can be expanded to include additional data, e.g., keys for keyed data structures.

\subsection{Verification}

To justify the translation into the array-based representation is an exercise in compiler verification. Our approach uses decompilation into logic. We also want to join the compiler verification results with properties of individual programs. For example, programs over recursive types in embedded systems are typically tail recursive, in order to avoid memory allocation. However, tail recursive programs (and their 


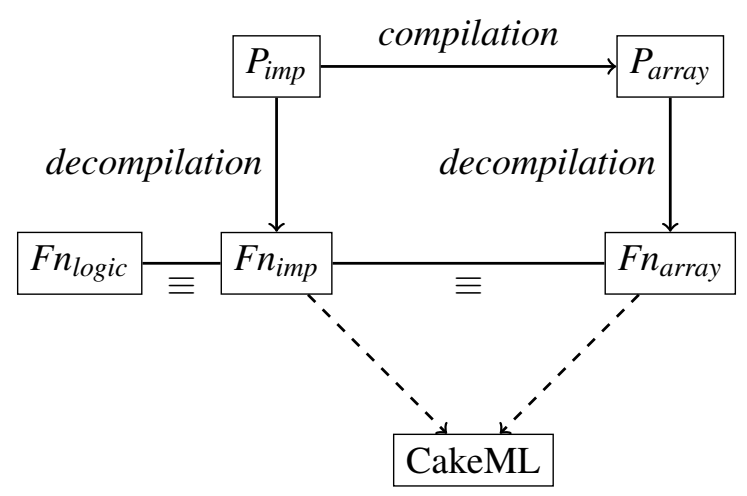

Figure 4: Verifying translation.

notorious while-loop brethren) are harder to reason about than recursive versions. So it is desirable to also have a high-level logical characterization of the program to reason about, provided properties proved at that level can be transported to the program representations.

Figure 4 illustrates the relationships: imperative program $P_{i m p}$ over tree-structured data is compiled to program $P_{\text {array }}$ over our array-based representation. These are decompiled to $F n_{\text {imp }}$ and $F n_{\text {array }}$, respectively. In our design, the formal equivalence between $F n_{\text {imp }}$ and $F n_{\text {array }}$ is intended to be automatically proved. The tactics for this are in development. On the other hand, the relationship between high-level logical function $F n_{\text {logic }}$ and $F n_{\text {imp }}$ may require ingenuity to show. The end result is that properties proved of $F n_{\text {logic }}$ can be transported - by use of the equivalence theorems - so that they apply, modulo adjustments to the underlying representation, to the evaluation of $P_{\text {array }}$ on the array representation.

Another interesting point is that the translation from $P_{i m p}$ to $P_{\text {array }}$ is informal, i.e., compilation is not achieved by proof steps, and yet decompilation into logic still allows a formal relationship between $P_{\text {imp }}$ and $P_{\text {array }}$ to be shown.

\section{Graphs}

The support for imperative functional programming via compilation into the array-based representation offers a useful level of abstraction for many programs. However, there are important applications where the full expressiveness of graphs is needed, for example route planning. In such cases, graphs could certainly be encoded via datatypes, but it is much more appealing to provide a direct representation. Therefore we have added graph constructs as primitives in DASL.

In particular, the declaration

$$
\text { graphtype name (nodeLabel, edgeLabel) }
$$

creates a new type name with the specified types of node labels and edge labels. With that declaration, an API for creating, traversing, and updating graphs of type name becomes available to the programmer. To exactly specify bounds on the numbers of nodes and edges, the sized declaration is extended, thus

$$
\text { sized theGraph : name }(m, n)
$$

declares a global variable theGraph holding an empty graph of type name with at most $m$ nodes, where each node can have at most $n$ outgoing edges. 
As an example of the use of DASL to implement the sorts of graph algorithms commonly encountered in autonomy and other high-assurance applications, consider the code for depth-first search depicted in Figure 5. The type of graphs handled by this code has unsigned integers at nodes (vertices) and unsigned integers labelling edges; other vertex and edge label types could just as easily been declared. Constants used to bound the graph are also declared. Finally, a fine point of the design is whether to expose the type of vertices. Since vertices are used to index into various data structures, we do show the representation.

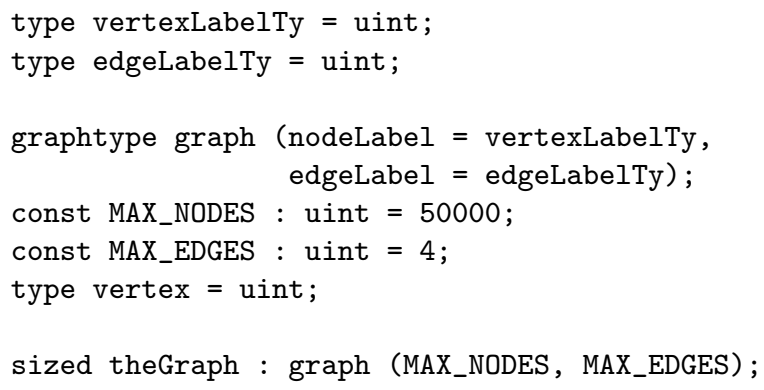

In this variant of depth-first search, we capture the spanning tree of the depth-first search as we proceed from a starting vertex. We use a binary search tree (BST) of key/value pairs to capture the spanning tree as the depth-first search proceeds, where the keys are the reached vertices, and the values are the immediately preceding vertices for the reached vertices. The BST for the spanning tree serves a dual purpose, as it is also used to check (via the exists function) whether a given vertex has been previously encountered during the current search, so that we can cut off search to already-explored sub-graphs.

The search function DFS_span is a recursive function whose parameters include a target vertex to be searched for (target); the graph $G$; the emerging spanning_tree of type BST; and a current fringe of unexplored edges, represented as a list of (vertex, predecessor-vertex) pairs (this list is conceptually a stack, and could be implemented using a defined DASL stack datatype). We also implement a "driver" function that initializes data structures, defines the initial vertex for the search, etc.; this driver is not shown due to space limitations.

The depth-first search in DFS_span proceeds as follows. If there are no fringe pairs to consider, we are done, and the (empty) fringe and spanning tree are returned to the caller. If the target vertex is in the spanning_tree, the search is successful, and we return the fringe and spanning tree to the caller. If there are fringe vertices to explore, we remove a $(v, v p r e d)$ pair from the front of the fringe list (using the $r s t$ rest-of-list function), then check to see if the vertex $v$ has already been encountered. If so, we are done with this fringe pair, and so we call DFS_span recursively to access the next fringe pair in the list (assuming there are any other pairs left). If $v$ has not been encountered before, we add the ( $v$, vpred) key/value pair to the spanning_tree, add children nodes of $v$ to the fringe list, utilizing the explore function (not shown), then call DFS_span to continue the search.

This algorithm statement is compact, and (in our-not-so-objective opinion) quite elegant. The dual use of the binary search tree for both marking visited vertices and recording the spanning tree as the algorithm proceeds is especially satisfying. Note also that a breadth-first search implementation can be readily obtained from the above by changing the explore function slightly, adding new fringe vertex pairs to the tail of the fringe list, rather than to the head of the list. Note also that a complete spanning tree from a given vertex can be obtained by providing a "bogus" target vertex that doesn't actually exist in the graph (we generally utilize the "null" vertex value of 0 for this purpose). Finally, the algorithm scales to millions of vertices, with tens of edges per vertex, executes quite quickly, and is readily compiled to hardware for even more speed. 


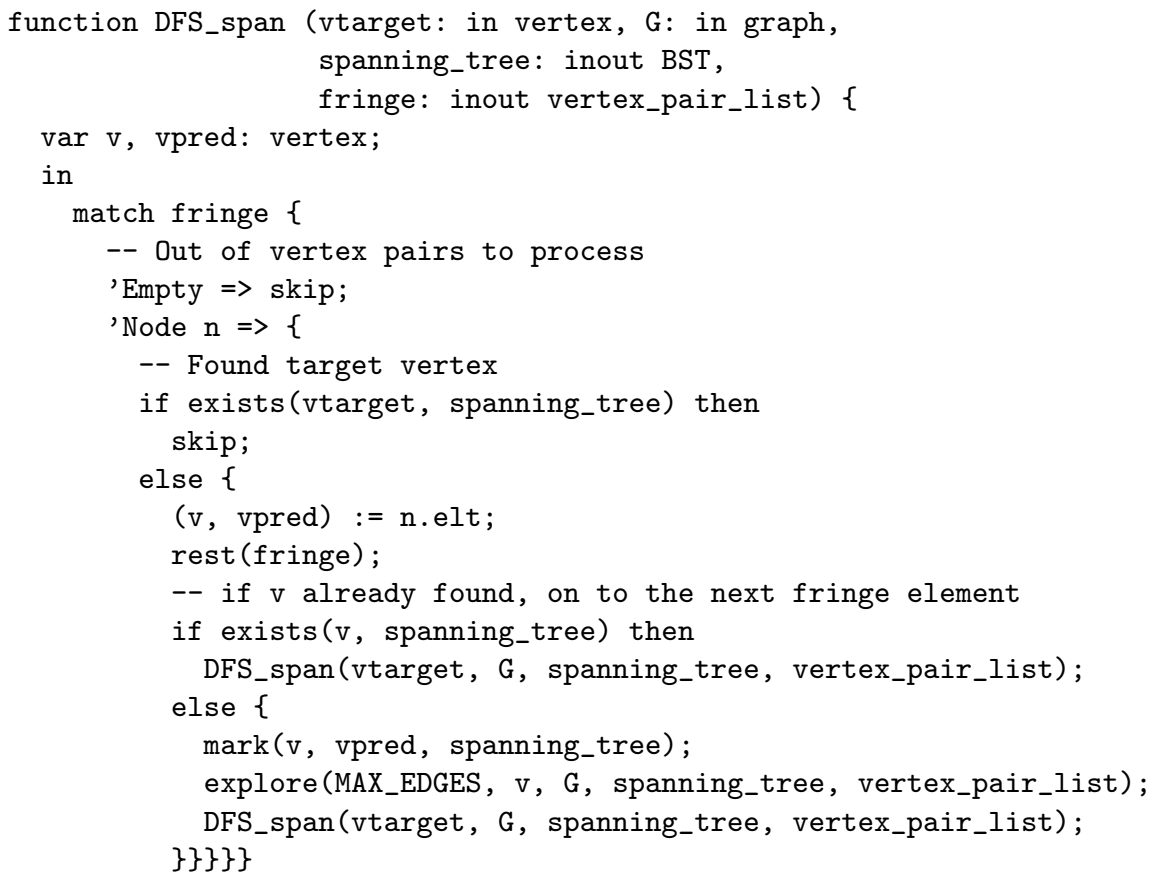

Figure 5: Depth-First Search in DASL.

\section{Use of ACL2 in DASL datatype and graphtype Design}

The development of the DASL language, toolchain, and runtime is a complex undertaking, and requires a solid foundation in the form of the basic datatype and graphtype design and implementation. Early on, we identified the need for a rapid, yet formal, prototyping environment that would allow us to experiment with design alternatives, evaluate these alternatives at scale, and provide initial proofs of correctness for data structure implementations before committing to a final design. Otherwise, we risked proceeding with flawed representations, requiring significant reworking of the DASL toolchain to repair. In short, we needed a "Semantic Laboratory" for DASL development. ACL2 filled the bill admirably:

- ACL2 is the most capable system we know of for the creation, proof, and execution of formal specifications. Using ACL2, we have been able to easily scale our data structure prototype implementations to millions of vertices and edges. We have been able to quickly execute algorithms to exercise these large data structures on concrete test input values, often generated at random, and validate the results of said algorithms operating on our prototype datatypes and graphtypes.

- ACL2 provides single-threaded objects, or stobjs, that provide functional data structure definitions with destructive "under-the-hood" implementations (subject to basic syntactic restrictions that guarantee that no "old" versions of mutated data structures exist in a given function). Thus, large data structures are not constantly being copied, as they would be for most pure functional implementations, eliminating garbage generation/collection times for stobj data structures. The contents of a stobj data structure also persist in the ACL2 world between events, providing convenient "near-global" variables that can be examined between function invocations, thus greatly aiding the debugging process. 
- ACL2 provides sophisticated proof libraries (books) for reasoning about aggregate data structures of arbitrary size, as well as fixed-size integers of various widths.

- Tail recursion in ACL2 combines recursive functional style with efficient compilation to loops.

- ACL2 guards promote a type-like discipline with the added rigor of formal proof. In particular, when we began writing data structure mutators, it became obvious that, in order to call said mutators from other guard-enabled functions, we would first need to prove that the mutators preserved the basic data structure "footprint" predicate provided by the defstobj event.

- ACL2's simple packaging facility provides separate namespaces for datatypes/graphtypes.

- All functions admitted to ACL2 must first be proven to terminate. This encourages the ACL2 developer to explicitly consider termination issues when writing functions.

- ACL2 is a mostly-automated theorem prover, and is quite adept at automated inductive proofs.

Thus, we implemented a Rudimentary ACL2 Semantic Laboratory for DASL, which we cheekily refer to as RASL DASL.

Of course, not all of these ACL2 features work together without issue. For example, it is more difficult to perform proofs about tail-recursive functions than their non-tail-recursive counterparts. Guards are not part of the ACL2 logic; if one wants the benefit of guard predicates in a function, one must restate those predicates in the function body. Not all ACL2 books work well in concert. Finally, stobjs are more difficult to reason about than, say, simple Lisp lists. Indeed, when we first starting using tail recursion and stobjs to implement "classic" data structures, we were largely frustrated in our efforts to perform proofs about compositions of functions operating on stobj-based data structures [8]. As often happens with ACL2, however, improvements in ACL2 over time, coupled with ACL2's uncanny ability to instruct the user to produce the kinds of forms that ACL2 "likes", have overcome many of these hurdles. We are now able to routinely obtain correctness proofs for compositions of tail-recursive functions operating on our prototype DASL "in-place" data structures using stobjs, albeit with some user/prover interaction.

\subsection{Example datatype: Binary Search Tree}

As an example of the ACL2-based datatype prototyping effort, consider the development of a basic Binary Search Tree (BST) datatype. The ACL2 stobj definition for this type is given in Figure6. (Note that graphtypes are defined similarly, as the underlying data structure representation is explicitly designed to represent graphs.) The declaration of the $\mathrm{Obj}$ stobj is followed by a number of basic functions (adding a vertex, deleting a vertex, etc. - not shown due to space constraints), as well as defthm forms that provide basic lemmas about the components of the datatype (e.g., that the key array is an integer-listp, or that the result of updating the val array continues to satisfy the Objp predicate that is synthesized by the defstobj form depicted in Figure 6.

In DASL datatype/graphtype prototyping, we generally place the basic structure definitions, etc. in one file, and the higher-level functions and theorems (e.g., functions that insert a new key/value pair, delete a key/value pair, check to see if a key is in the BST, return the value for a given key, and so on) in a second file. That way, similar datatypes that share a common structure can reuse the file of basic definitions, with slight modifications. As an example of the sort of higher-level functions we typically define, consider the getVal function of Figure 7, along with its shorthand macro form getV. getVal follows the classic textbook definition, with a bit of the underlying ACL2 data representation showing through. Note that termination for this function is not assured in general; thus, we add a count parameter that is decremented on each recursive call of getVal. Fortunately, we have a convenient value at hand to serve as a reasonable initial value for count, namely the number of vertices in the BST. 


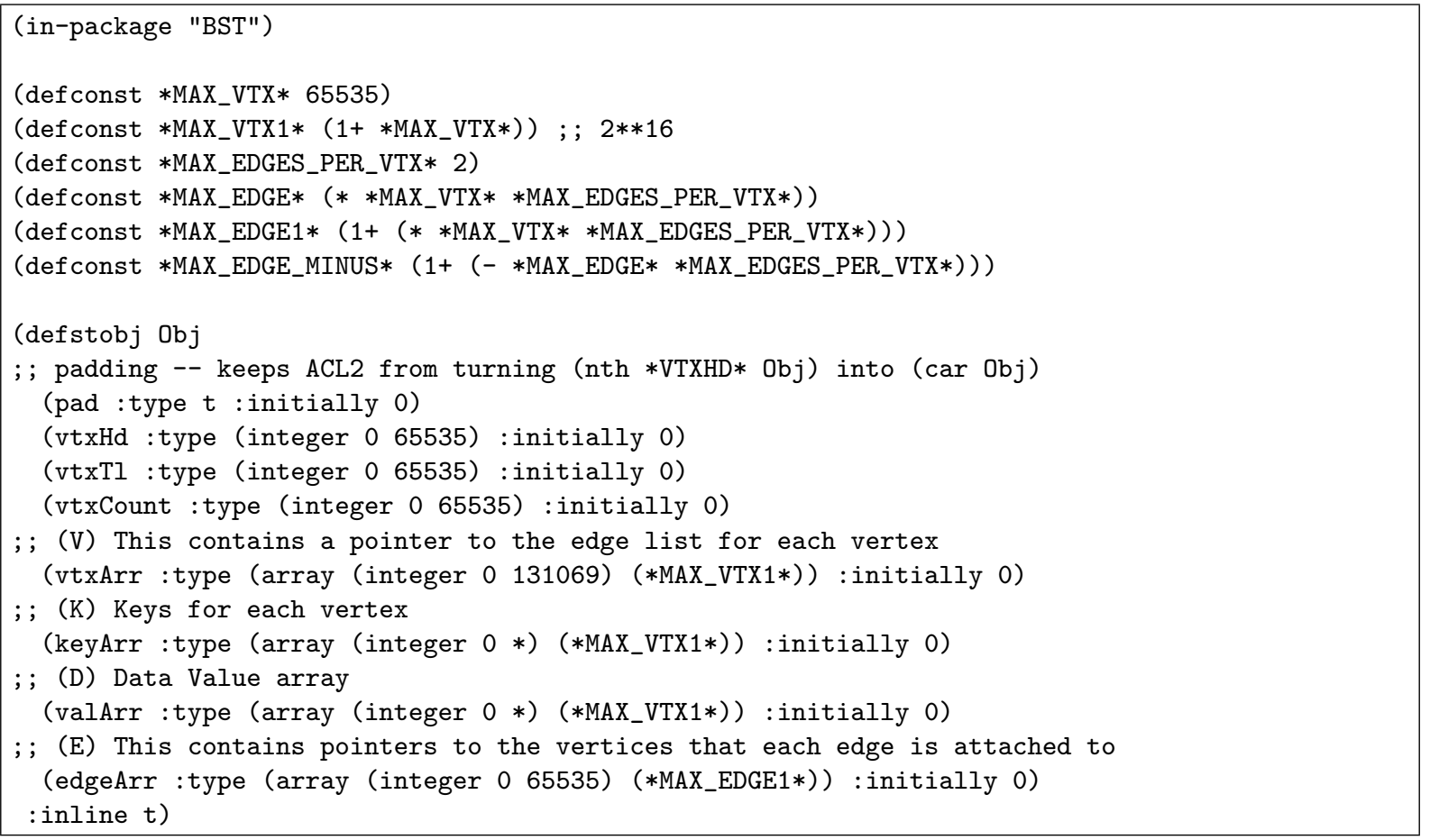

Figure 6: Binary Search Tree stobj declaration.

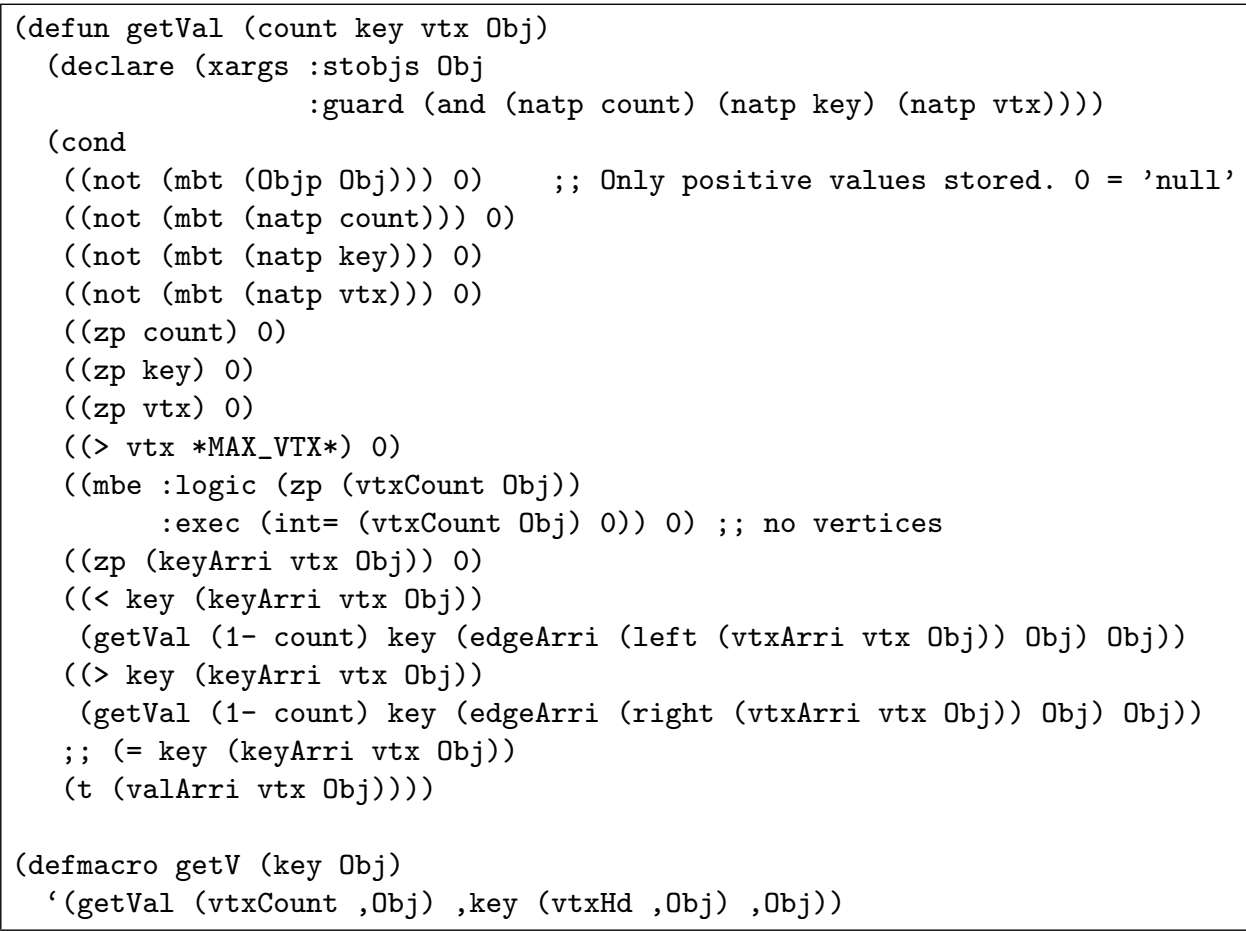

Figure 7: Example DASL-derived Binary Search Tree function prototyped in ACL2. 


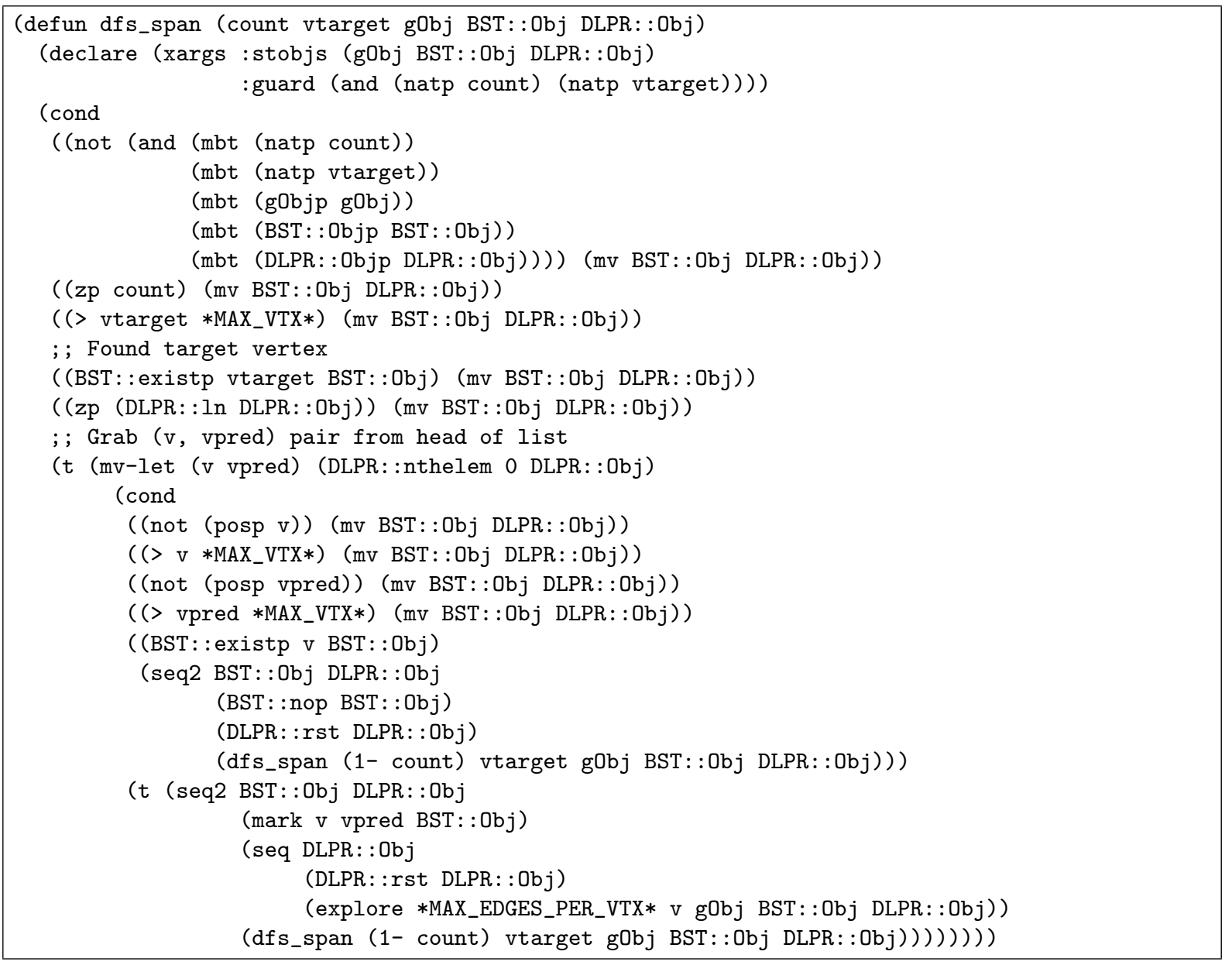

Figure 8: Depth-First Search prototyped in ACL2.

\section{Results}

As an example of the sorts of algorithms we are able to successfully prototype using the RASL DASL environment, consider the hand-translated ACL2 version of the Depth-First Search algorithm of Figure 5 depicted in Figure 8. The ACL2 version utilizes a graphtype (gObj, taking on the role of the DASL G parameter) and two datatypes: the Binary Search Tree previously discussed (BST::Obj, corresponding to the DASL spanning_tree parameter), and a doubly-linked list (DLST::Obj, acting as the DASL fringe parameter). We employ a doubly-linked list, which provides access/update from either end of the list, for the fringe parameter because it was convenient to also use the same datatype for the implementation of the Breadth-FIrst Search algorithm (in fact, dfs_span and bf s_span are functionally identical; the difference in behavior between BFS and DFS is solely due to whether the explore function adds the vertex pairs it finds to the front (DFS) or back (BFS) of the list.

As in the BST getVal function, the termination of the df s_span function is not explicitly assured, so we again add a count parameter that is decremented with each recursive call. The number of vertices in the graph multiplied by the maximum number of edges per vertex serves as a reasonable initial value for count. Another unique feature of the ACL2 code requiring some explanation is the seq2 macro. 
We often use J Moore's seq macro when working with stobjs, as it eliminates much of the let binding "clutter", allowing one to simply write one stobj-manipulating expression after another within the scope of the seq. With two stobjs to update and return, the mv-let binding and mv return "clutter" can get much worse. Thus, we developed a quick-and-dirty macro, seq2 to do the analogous decluttering job for two stobjs. seq2 takes as parameters the two stobjs that are to be updated, the updating s-expression for the first stobj, the updating s-expression for the second stobj, and the s-expression that one wishes to return using mv. This works well enough in practice, although sometimes only one of the two stobjs one wishes to return using mv are actually modified within the scope of the seq2. In this case, one must provide some "no-op" s-expression for the unmodified stobj.

Other than these few changes, and a slight rearrangement to concentrate the stobj updates into one contiguous section of the code, the ACL2 version tracks the DASL code fairly well, and the elegance of the algorithm is still apparent.

To date, we have written a number of autonomy-relevant algorithms in DASL after prototyping in RASL DASL, including tree search, graph search, Dijkstra's all-pairs shortest path algorithm, and unify/substitute; as well as a number of supporting data structures, such as priority queues, stacks, singly- and doubly-linked lists, queues, deques, etc. We have used our verification environment to state and prove properties of many of these algorithms, starting with basic well-formedness, and proceeding to full functional correctness. Finally, we have generated code for many of these examples using the DASL compiler, and have validated the code generation via testing. As expected, the array-based form leads to efficient execution, scales well to millions of vertices with tens of edges per vertex, and enables hardware-based and GPU-based execution.

\section{Related Work and Future Work}

We first acknowledge the influence that ACL2 single-threaded objects [3] have had on the overall DASL philosophical approach of providing functional specifications with efficient, "under-the-hood" implementations. We have learned much from the ACL2 developers, particularly the importance of efficient, executable formal specifications.

The Boogie [17] and WhyML/Why3 [5] systems are paradigmatic IVLs, supporting highly developed verification-enhanced languages. The main point of departure with our work is that Boogie and WhyML are programming languages, while the IVL for DASL is higher order logic. Verification-enhanced versions of C are supported by Boogie and Why3, and also by Appel's Hoare Logic for CompCert C [1], which is derived from the operational semantics of CompCert in Coq. The AutoCorres tool [6] arose out of the seL4.verified effort; it translates ASTs from a parser for the C dialect used in seL4 to Schirmer's SIMPL theory in Isabelle/HOL and helps automate much of the Separation Logic used.

Chlipala's Bedrock system [4] also utilizes higher order logic as an IVL; in particular, he uses Coq as the substrate on which to build intermediate and lower-level languages and prove the correctness of transformations. Finally, the work of O'Leary and Russinoff on the formalization of C subsets for hardware design in ACL2 [22] encouraged us to consider a formalized System language.

DASL is a direct descendent of the Guardol Domain-Specific Language for Cross-Domain Systems [12]. Our experience with Guardol convinced us of the efficacy of taking a Domain-Aware approach to language development for various high-assurance domains. Not surprisingly, DASL and Guardol share a number of syntactic and semantic features. DASL also shares a great deal of the verification-oriented tool infrastructure pioneered on the Guardol effort, such as the logic-based IVL, the RADA backend tree solver, as well a VHDL code generator. The latter capability allowed us to synthesize a formally proven 
high-level regular expression pattern matcher in inexpensive FPGA hardware that performed at Gigabit Ethernet line speeds [11]; these results prompted us to continue to develop VHDL code generation for DASL. DASL distinguishes itself from Guardol mainly in the datatype and graphtype declarations, the sized declaration, and the attendant syntactic restrictions that allow for compilation to an efficient in-place data structure representation.

In future work, we will continue to develop the DASL toolchain, focusing on code generation for CakeML, but also improving code generation for Ada, Java, VHDL, and other target languages. We will also continue to refine the language syntax, focusing on ways to conveniently add extensions such as grammar specifications and rulebases. Regular expressions are introduced currently by way of a simple regex_match intrinsic function, which accepts a regular expression as a string parameter, but this method is unlikely to scale well to grammar rules, etc. Finally, we will continue to expand on the suite of applications implemented in DASL, especially in the areas of autonomy algorithms, as well as data filtering/transformation for cyber-resilient systems.

\section{Conclusion}

We have developed a verifying compilation technique for a domain-aware programming language for autonomy and other high-assurance applications that combines efficient implementations of high-level data structures used in autonomous systems with the high assurance needed for accreditation. Our system supports a "natural" functional proof style, yet applies to more efficient data structure implementations. Our toolchain features code generation to mainstream programming languages, as well as GPU-based and hardware-based realizations. We base the Intermediate Verification Language for our toolchain upon higher-order logic. By giving program execution a formal semantics, claims about program behavior can be mathematically proven, and source-to-source transformations of the intermediate form can be proven as well. Thus, proofs about high-level programs over high-level data structures can be carried out while automatically ensuring a formal, proved connection to the low-level efficient implementation. We have also demonstrated that when the IVL is higher order logic, verified code generation is possible via the facilities of the CakeML verified compiler.

We utilized ACL2 to develop our efficient yet verifiable data structure design. ACL2 is particularly well-suited for this task, with its sophisticated libraries for reasoning about aggregate data structures of arbitrary size, efficient execution of formal specifications, and its support for single-threaded objects, not to mention its strength as an automated inductive prover. We described our high-assurance data structure design approach in ACL2, presented ACL2 examples of common algebraic data types implemented using this design approach, discussed proofs of correctness for those data types carried out in ACL2, as well as sample ACL2 implementations of relevant algorithms that utilize these efficient, high-assurance data structures. In summary, this ACL2-based development activity has produced a performant, as well as highly-assured, data structure design for critical applications, and we continue to use this ACL2-based design environment to prototype new applications for modern high-assurance systems, such as lexers and parsers for data interchange formats such as JSON, inference engines, and the like.

\section{Acknowledgments}

We thank the anonymous referees for their helpful comments. This work was sponsored in part by the Defense Advanced Research Projects Agency (DARPA). 


\section{References}

[1] Andrew W. Appel (2014): Program Logics for Certified Compilers. Cambridge University Press, doi $10.1017 /$ CBO9781107256552

[2] Jason Belt, John Hatcliff, Robby, Patrice Chalin, David S. Hardin \& Xianghua Deng (2011): Bakar Kiasan: Flexible Contract Checking for Critical Systems using Symbolic Execution. In: Proceedings of the Third NASA Formal Methods Symposium (NFM 2011), pp. 58 - 72, doi:10.1007/978-3-642-20398-5_6

[3] Robert S. Boyer \& J Strother Moore (2002): Single-Threaded Objects in ACL2. In: Practical Aspects of Declarative Languages, 4th International Symposium, PADL 2002, LNCS 2257, Springer, pp. 9-27, doi $10.1007 / 3-540-45587-6 \_3$.

[4] Adam Chlipala (2013): The Bedrock Structured Programming System: Combining Generative Metaprogramming and Hoare Logic in an Extensible Program Verifier. In: Proceedings of the 18th ACM SIGPLAN International Conference on Functional Programming, ICFP '13, ACM Press, pp. 391-402, doi $10.1145 / 2500365.2500592$

[5] Jean-Christophe Filliâtre \& Andrei Paskevich (2013): Why3 - Where Programs Meet Provers. In Matthias Felleisen \& Philippa Gardner, editors: Proceedings of the 22nd European Symposium on Programming, LNCS 7792, Springer, pp. 125-128, doi: 10.1007/978-3-642-37036-6_8.

[6] David Greenaway, Japheth Lim, June Andronick \& Gerwin Klein (2014): Don't Sweat the Small Stuff: Formal Verification of $C$ Code Without the Pain. In: Proceedings of the 35th ACM SIGPLAN Conference on Programming Language Design and Implementation, PLDI '14, ACM Press, pp. 429-439, doi $10.1145 / 2594291.2594296$

[7] David S. Hardin, editor (2010): Design and Verification of Microprocessor Systems for High-Assurance Applications. Springer, doi $10.1007 / 978-1-4419-1539-9$.

[8] David S. Hardin \& Samuel S. Hardin (2009): Efficient, Formally Verifiable Data Structures using ACL2 Single-Threaded Objects for High-Assurance Systems. In S. Ray \& D. Russinoff, editors: Proceedings of the Eighth International Workshop on the ACL2 Theorem Prover and its Applications, ACM Press, pp. 100 105, doi $10.1145 / 1637837.1637853$.

[9] David S. Hardin \& Samuel S. Hardin (2013): ACL2 Meets the GPU: Formalizing a CUDA-based Parallelizable All-Pairs Shortest Path Algorithm in ACL2. In R. Gamboa \& J. Davis, editors: Proceedings of the 11th International Workshop on the ACL2 Theorem Prover and its Applications, 114, EPTCS, pp. 127 - 142, doi 10.4204/EPTCS.114.10

[10] David S. Hardin, T. Douglas Hiratzka, D. Randolph Johnson, Lucas G. Wagner \& Michael W. Whalen (2009): Development of Security Software: A High Assurance Methodology. In K. Breitman \& A. Cavalcanti, editors: Proceedings of the 11th International Conference on Formal Engineering Methods: Formal Methods and Software Engineering (ICFEM'09), Springer, pp. 266 - 285, doi:10.1007/978-3-642-10373-5_14.

[11] David S. Hardin, Konrad L. Slind, Mark A. Bortz, James Potts \& Scott Owens (2016): A High-Assurance, High-Performance Hardware-Based Cross-Domain System. In: Computer Safety, Reliability, and Security 35th International Conference, SAFECOMP 2016, LNCS 9922, Springer, pp. 102-113, doi 10.1007/978-3319-45477-1_9.

[12] David S. Hardin, Konrad L. Slind, Michael W. Whalen \& Tuan-Hung Pham (2012): The Guardol Language and Verification System. In: Proceedings of TACAS, LNCS 7214, Springer, pp. 18-32, doi 10.1007/978-3642-28756-5_3.

[13] Parwan Harish \& P.J. Narayanan (2007): Accelerating Large Graph Algorithms on the GPU using CUDA. In: IEEE High Performance Computing - HiPC 2007, LNCS 4873, Springer, pp. 197-208, doi 10.1007/9783-540-77220-0_21.

[14] Matt Kaufmann, Panagiotis Manolios \& J Strother Moore (2000): Computer-Aided Reasoning: An Approach. Texts and Monographs in Computer Science, Kluwer Academic Publishers, doi 10.1007/978-1-4615-4449-4. 
[15] Ramana Kumar, Magnus O. Myreen, Michael Norrish \& Scott Owens (2014): CakeML: A Verified Implementation of ML. In: POPL '14: Proceedings of the 41st ACM SIGPLAN-SIGACT Symposium on Principles of Programming Languages, ACM Press, pp. 179-191, doi:10.1145/2535838.2535841.

[16] K. Rustan M. Leino (2013): Developing Verified Programs with Dafny. In: Proceedings of the 2013 International Conference on Software Engineering, ICSE '13, IEEE Press, pp. 14881490, doi:10.1109/icse.2013.6606754 Available at http://dl.acm.org/citation.cfm?id=2486788. 2487050.

[17] K. Rustan M. Leino \& P. Ruemmer (2010): A Polymorphic Intermediate Verification Language: Design and Logical Encoding. In: Proceedings of TACAS, LNCS 6015, pp. 312-327, doi:10.1007/978-3-642-12002-2_26.

[18] Xavier Leroy (2009): Formal verification of a realistic compiler. Communications of the ACM 52(7), pp. 107-115, doi:10.1145/1538788.1538814.

[19] John W McCormick \& Peter C. Chapin (2015): Building High Integrity Applications with SPARK. Cambridge University Press, doi:10.1017/cbo9781139629294.

[20] Magnus Myreen (2009): Formal verification of machine-code programs. Ph.D. thesis, University of Cambridge.

[21] Magnus O. Myreen \& Scott Owens (2014): Proof-producing Translation of Higher-order logic into Pure and Stateful ML. Journal of Functional Programming 24(2-3), pp. 284-315, doi:10.1017/S0956796813000282.

[22] John W. O'Leary \& David M. Russinoff (2014): Modeling Algorithms in SystemC and ACL2. In: Proceedings of the 12th International Workshop on the ACL2 Theorem Prover and its Applications, 152, EPTCS, pp. 145162, doi 10.4204/EPTCS.152.12 Available at https://arxiv.org/pdf/1406.1565.pdf

[23] Tuan-Hung Pham (2014): Verification of Recursive Data Types using Abstractions. Ph.D. thesis, Department of Computer Science and Engineering, University of Minnesota.

[24] RTCA Committee SC-205 (2015): DO-178C Software Considerations in Airborne Systems and Equipment Certification. Available at https://my.rtca.org/nc__store?search=DO-178C.

[25] Norbert Schirmer (2006): Verification of sequential imperative programs in Isabelle/HOL. Ph.D. thesis, TU Munich.

[26] Andrew Tolmach \& Dino P. Oliva (1998): From ML to Ada: Strongly-typed Language Interoperability via Source Translation. Journal of Functional Programming 8(4), pp. 367 - 412, doi:10.1017/s0956796898003086.

[27] Harvey Tuch, Gerwin Klein \& Michael Norrish (2007): Types, Bytes, and Separation Logic. In: Proceedings of the 34th Annual ACM SIGPLAN-SIGACT Symposium on Principles of Programming Languages, POPL '07, ACM Press, pp. 97-108, doi $10.1145 / 1190216.1190234$. 